\title{
BMJ Open Pregnancy-related venous thrombosis: comparison between spontaneous and ART conception in an Italian cohort
}

\author{
Michela Villani, ${ }^{1}$ Francesco Dentali, ${ }^{2}$ Donatella Colaizzo, ${ }^{1}$ Giovanni Luca Tiscia, ${ }^{1}$ \\ Patrizia Vergura, ${ }^{1}$ Tiziana Petruccelli, ${ }^{1}$ Francesco Petruzzelli, ${ }^{3}$ Walter Ageno, ${ }^{2}$ \\ Maurizio Margaglione, ${ }^{4}$ Elvira Grandone ${ }^{1}$
}

To cite: Villani M, Dentali F, Colaizzo D, et al. Pregnancyrelated venous thrombosis: comparison between spontaneous and ART conception in an Italian cohort. BMJ Open 2015;5: e008213. doi:10.1136/ bmjopen-2015-008213

- Prepublication history for this paper is available online. To view these files please visit the journal online (http://dx.doi.org/10.1136/ bmjopen-2015-008213).

Received 17 March 2015 Revised 28 June 2015 Accepted 17 July 2015

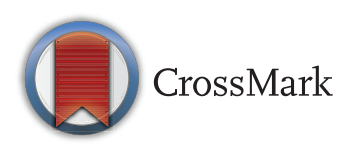

${ }^{1}$ Atherosclerosis and Thrombosis Unit, I.R.C.C.S. "Casa Sollievo della Sofferenza", Foggia, Italy 2Department of Clinical Medicine, Insubria University, Varese, Italy

${ }^{3}$ Obstetrics and Gynaecology Department, I.R.C.C.S. "Casa Sollievo della Sofferenza", Foggia, Italy

${ }^{4}$ Department of Medical Genetics, University of Foggia, Foggia, Italy

Correspondence to Dr Elvira Grandone; e.grandone@operapadrepio.it

\section{ABSTRACT}

Objective: To evaluate in an Italian cohort the incidence of venous thromboembolic events (VTE) in pregnancies after assisted reproductive technologies (ART).

Setting: Thrombosis and Haemostasis Unit at I.R.C.C.S. 'Casa Sollievo della Sofferenza', S. Giovanni Rotondo.

Participants: A prospective cohort of 998 women advised to undergo ART was referred by local fertility clinics from April 2002 to July 2011. Follow-up information was obtained during the check-up and/or by phone interviews. In a cohort of women who consecutively gave birth $(n=3339)$ after spontaneous conception in our Institution, information on the diagnoses of pregnancy-related venous thromboses was obtained by linkage to a patient administrative register.

Primary and secondary outcome measures: We calculated the incidence of VTE and superficial venous thrombosis in successful ART cycles and compared it with that of the general population conceiving spontaneously.

Results: Overall, 684 ART cycles were carried out by 234 women, who achieved a clinical pregnancy; in case of more than one successful cycle, only the first pregnancy was considered. Three vein thromboses (two VTE and one superficial vein thrombosis) were recorded. An antithrombotic prophylaxis with LMWH alone or combined with low-dose aspirin was prescribed in 23/234 (9.8\%) women. In the reference cohort of 3339 women, a total of 11 vein thromboses were observed: six VTE and five SVT. The two-tailed Fisher exact test showed a trend towards statistical significance ( $p$ : 0.06 , OR: $3.9,95 \% \mathrm{Cl} 0.87$ to 15.3 ). After the exclusion of superficial thromboses in both the groups, we found that the incidence of VTE in our population of women who had undergone ART was 2/234 pregnancies $(8.5 \%$ o), whereas that in our reference population was $6 / 3339(1.8 \%$ \%) (p: 0.09).

Conclusions: Our data show a slightly higher incidence of vein thromboses in pregnancies after ART than in those after natural conception.

\section{INTRODUCTION}

It is well known that pro-coagulant changes occurring during normal pregnancy cause

\section{Strengths and limitations of this study}

- This study was carried out in a single Institution of the Apulia region. Cases were referred by Fertility Clinics of the same region.

- The reference cohort of pregnant women who delivered at the same Institution was representative of the general population from the same geographical area, as maternal age and the number of live births were comparable in the two populations.

- In the reference cohort, the number of vein thromboses may be under-represented in respect of the general pregnant population, as some cases may not be admitted to the hospital.

- We were not able to evaluate in the reference cohort the influence of other risk factors and, in the study group, that of change in assisted reproductive technologies practice during a rather long period (2002-2011).

an increased risk of venous thromboembolism (VTE). Previous studies have found that VTE after a spontaneous conception is slightly more than 1 of 1000 pregnant women. ${ }^{1}{ }^{2}$ In women undergoing assisted reproductive technologies (ART), medical ovarian stimulation is thought to increase thrombotic risk. However, available data about the magnitude and the duration of VTE risk in ART are conflicting. A review of several published case reports concluded that in in-vitro fertilisation (IVF) the VTE risk is comparable to that of spontaneous conceptions. ${ }^{3}$ Similarly, a more recent Danish register-based cohort study did not find an increased risk after IVF treatment in unsuccessful cycles. ${ }^{4}$ Nevertheless, an increased risk in successful IVF cycles was very recently reported, and the risk seems to be significantly higher during the first trimester. ${ }^{4-7}$ An important role as a risk factor for VTE is played by ovarian hyperstimulation syndrome (OHSS) occurring during and 
after an ART procedure, with supraphysiological oestradiol levels that sometimes can result. During ovarian stimulation, the coagulation and fibrinolytic systems are activated; this activation appears to be exaggerated and prolonged with the development of OHSS. ${ }^{8}$ This syndrome is a systemic disorder resulting from vasoactive products released by hyperstimulated ovaries. Haemoconcentration, altered coagulation system and reduced venous return secondary to enlarged ovaries, ascites and immobility are likely to be important determinants for the increased risk of venous thrombosis in women with OHSS. Thrombotic events have also been reported to occur weeks after OHSS has resolved. ${ }^{9}$ It has been calculated that OHSS increases until 100-fold the risk of VTE. ${ }^{6}$ Inherited or acquired thrombophilias, body mass index (BMI), increasing age and reduced mobility could further increase this risk. A previous large study carried out in our centre showed that age over 39 and mild hyperhomocysteinaemia are strongly associated with the occurrence of thrombotic events during ART, while the impact of common inherited causes of thrombophilia was not statistically relevant. ${ }^{10} \mathrm{~A}$ similar result was shown by Ricci $e t a l^{11}$ in a prospective cohort of nulliparous women undergone ART cycles: neither the presence of factor V Leiden (FVL) nor the FIIA20210 prothrombin mutation (PTm) gene variants influenced the thrombotic risk. In the present study, we prospectively evaluated the incidence of VTE in successful ART cycles and, for the first time in an Italian cohort, compared it with that of the general population conceiving spontaneously.

\section{PATIENTS AND METHODS}

We identified 998 women candidate to ART consecutively referred by local Fertility Clinics to our Unit from April 2002 to July 2011. At that time, detailed information on maternal age, BMI, smoking status, causes of infertility, personal history of obstetric complications and VTE was collected. All the women were investigated for the presence of inherited (FVL, PTm and deficiencies in protein $\mathrm{S}$ and $\mathrm{C}$ and antithrombin) and acquired (lupus anticoagulant (LA), anticardiolipin antibodies (aCL)) thrombophilias. Follow-up information was obtained during the check-up and/or by phone interviews. The prescription of aspirin and/or LMWHs was verified by reviewing medical records. For each woman, the following information was collected: cycles number, type of ART procedure (IVF and intracytoplasmatic sperm injection (ICSI), or intrauterine injection (IUI)), occurrence of clinical pregnancy (defined as the ultrasonographic visualisation of one or more gestational sacs and fetal heart beat), ${ }^{12}$ pregnancy loss (defined as a loss occurring before/at 20 weeks of gestation), intrauterine fetal death (a loss occurring after 20 weeks), live birth (the delivery of a live newborn), presence of OHSS, obstetric and thromboembolic complications. Diagnosis of VTE or superficial vein thrombosis (SVT) was objectively confirmed by Doppler ultrasonography, ventilation-perfusion lung scanning or pulmonary angiography. ${ }^{8}$ Only cycles starting with ovarian stimulation and ending with embryotransfer/IUI were considered.

We identified all women who had undergone at least one ART cycle and achieved at least one clinical pregnancy after the procedure. However, for final analysis, in case of more than one successful cycle, only the first pregnancy was considered. Similarly, in the reference cohort, when the same woman delivered more than once, only the first pregnancy was computed.

Between January 2010 and December 2012, 3527 women gave birth at our hospital; after the exclusion of 155 pregnancies of those who delivered in this period more than once and 33 pregnancies obtained by means of ART, 3339 pregnancies were considered as the reference group. From 2010, an electronic database is available in our Institution that includes validated information on the pregnancy, delivery and neonatal periods. We obtained information on the diagnoses of pregnancy-related VTE by linkage to our Institutional patient register, an administrative database containing information on the dates of admission and discharge and the main diagnosis. The choice to consider also SVT depends on the reported association with deep-vein thrombosis, as a high rate of pulmonary embolism in patients with saphenous vein thrombosis and no obvious deep vein involvement has been described. ${ }^{13}$

Diagnoses were recorded according to the International Classification of Diseases, 9th revision (ICD-9): codes 325, 415.1, 451, 452, 453, 671.3, 671.4, 671.5, 671.9, 673.2, 673.3. In order to evaluate whether our reference group was representative of the regional population, we compared median age and the number of live births of women who delivered at our Hospital and women who delivered in a similar time lapse (2008-2010) in the same Italian region (Apulia). This last information was extracted from the Italian birth register at the National Board of Health and Welfare. ${ }^{14}$ The study was approved by the local ethics committee, and participants gave their written informed consent for present and future use of clinical data.

\section{Laboratory tests}

Blood samples were collected in $3.8 \%$ trisodium citrate and centrifuged at $2000 \mathrm{~g}$ for $15 \mathrm{~min}$ to obtain plateletpoor plasma, which was frozen and stored in small aliquots at $-70^{\circ} \mathrm{C}$ until tested. Antiphospholipid antibodies-LA and IgG, IgM aCL (QUANTA Lite, INOVA Diagnostics, San Diego, California, USA) - antithrombin and protein $\mathrm{C}$ (Berichrom Antithrombin and Protein C amidolytic assays, Behring, Germany) and free protein $\mathrm{S}$ (IMUCLONE Protein S ELISA, American Diagnostica, Stamford, Connecticut, USA) were determined in all patients. A confirmed presence of antiphospholipid antibodies was defined according to SSC criteria, ${ }^{15}$ or the concurrent presence of more than one. DNA was 
extracted from peripheral blood leucocytes according to standard protocols.

FVL and PTm genotyping was performed by a TaqMan (Applied Biosystems, Foster City, California, USA) probe-based real-time PCR technique. ${ }^{16}$

\section{Statistical analysis}

All the analyses were performed using SPSS V.11.0 (SPSS Inc, Chicago, Illinois, USA). The significance of any difference in means was evaluated by non-parametric tests, whereas the significance of any difference in proportions was tested using the Fisher exact test or by $\chi^{2}$ statistics as appropriate. OR and 95\% CIs were calculated.

\section{RESULTS}

A flow chart of the study cohort is shown in figure 1 . Overall, we identified 234 women who achieved a clinical pregnancy after an ART cycle. Median BMI was $22.4 \mathrm{~kg} / \mathrm{m}^{2}$, most women did not smoke $(79.5 \%)$ and $10.3 \%$ carried congenital or acquired thrombophilias (table 1). As far as severe thrombophilias are concerned, two women showed protein S deficiency (one combined with FV Leiden) and one a confirmed presence of antiphospholipid antibodies. The frequencies we observed were not significantly different from those found in a general population from the same ethnic background. ${ }^{17}$

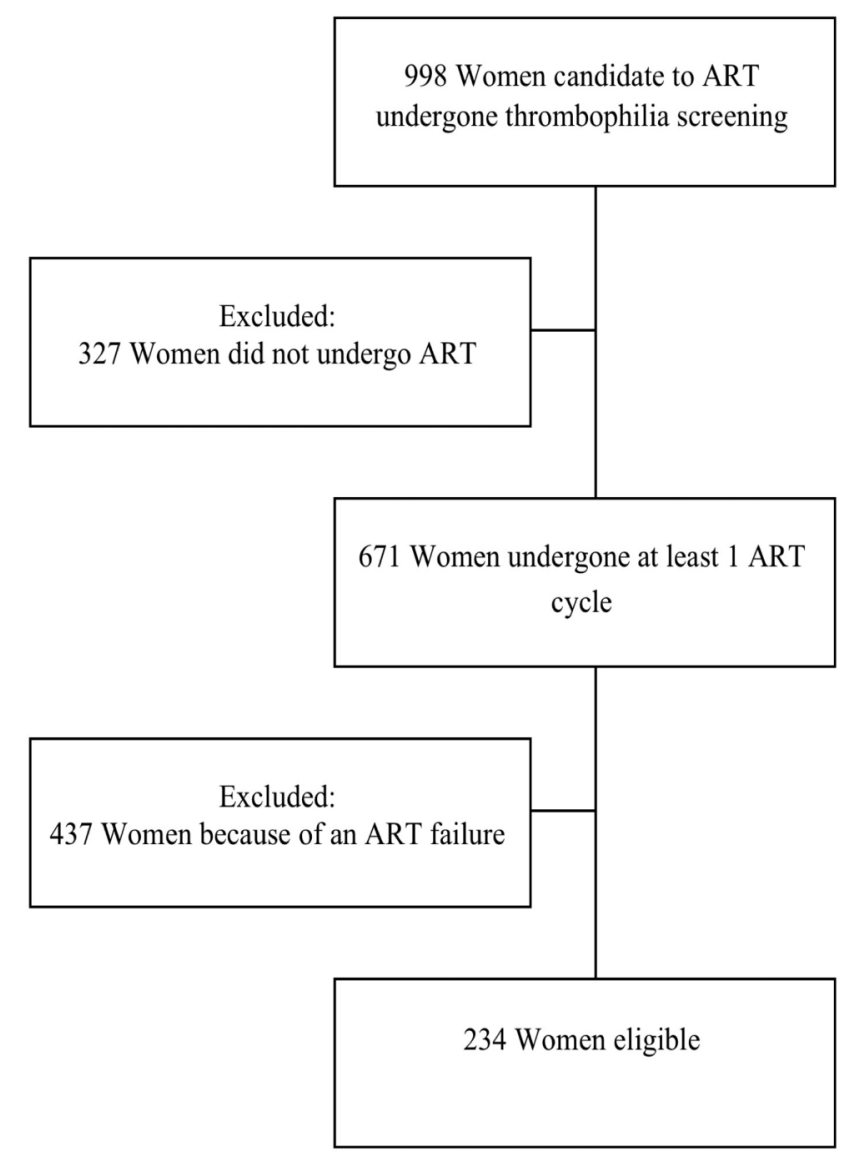

Figure 1 Study cohort (April 2002-July 2011). ART, assisted reproductive technologies.

\begin{tabular}{|c|c|}
\hline Age years, median (range) & $34(23-46)$ \\
\hline BMI, median (range) & $22.4(16.7-35.9)$ \\
\hline \multicolumn{2}{|l|}{ Smoking habits, n/N (\%) } \\
\hline Unknown & 7 (3) \\
\hline No smokers & $186(79.5)$ \\
\hline $1-10$ cigarettes per day & $31(13.2)$ \\
\hline 10-20 cigarettes per day & 7 (3) \\
\hline$>20$ cigarettes per day & $1(0.4)$ \\
\hline Not provided & $2(0.9)$ \\
\hline \multicolumn{2}{|l|}{ Infertility factors, n/N (\%) } \\
\hline Male & 92 (39.3) \\
\hline Female & $50(21.3)$ \\
\hline Unexplained & 79 (33.8) \\
\hline Mixed & $10(4.3)$ \\
\hline Unknown & $3(1.3)$ \\
\hline FVL, n/N (\%) & $11(4.7)$ \\
\hline PTm, n/N (\%) & $10(4.3)$ \\
\hline Severe thrombophilias ${ }^{*}, n / N(\%)$ & $3(1.3)$ \\
\hline
\end{tabular}

Overall, these women were exposed to a total of 684 ART cycles, 75 IUI and 609 IVF/ICSI with a clinical pregnancy rate of $33.3 \% \quad(n=25)$ and $41 \% \quad(n=250)$, respectively. Thirty-five women had more than one clinical pregnancy; in this case, we considered only the first pregnancy; thus, 234 clinical pregnancies were included in the final analysis. One hundred and forty-seven $(62.8 \%)$ pregnancies resulted in live births. Table 2 shows clinical features of women who suffered from VTE: two of three women tested negative for thrombophilia, while one had a PTm in heterozygosis; the latter had age and BMI as additional risk factors. None presented a family history of VTE. In one case, a SVT in the left leg was recorded during prophylaxis with LMWH in a patient suffering from erythema nodosum; later on, this patient also had a recurrence of SVT in the right leg in a following pregnancy during prophylaxis with LMWH. Both the events occurred during the first trimester.

An antithrombotic prophylaxis with LMWH alone or combined with low-dose aspirin was prescribed in 23/ 234 (9.8\%) women; among them, 8/23 (34.8\%) were thrombophilic (three FVL heterozygotes, three PTm heterozygotes, one carried a protein $\mathrm{S}$ deficiency combined with FVL and one a confirmed presence of antiphospholipid antibodies). Among non-treated women $(\mathrm{n}=211)$, 16 carried thrombophilia: 8 were FVL heterozygotes, $7 \mathrm{PTm}$ heterozygotes and 1 carried a protein S deficiency. When we compared the incidence of thrombotic events in the presence or absence of prophylaxis with LMWH, we found no significant difference between the groups (thromboses: $1 / 23$ vs $2 / 211$, p: ns). The median age of the whole group of cases was 34 years (23-46) and was not significantly different from those who were 
Table 2 Characteristics of women from the study cohort experiencing venous thrombosis

\begin{tabular}{|c|c|c|c|c|c|c|c|}
\hline Patients & $\begin{array}{l}\text { Age at } \\
\text { event }\end{array}$ & BMI & $\begin{array}{l}\text { Thrombophilia or } \\
\text { OHSS }\end{array}$ & $\begin{array}{l}\text { Additional } \\
\text { risk factors }\end{array}$ & Cycle & Type of event & $\begin{array}{l}\text { Antithrombotic } \\
\text { prophylaxis }\end{array}$ \\
\hline 1 & 30 & 22.3 & No & No & 1 & $\begin{array}{l}\text { SVT in the left leg at } 12 \text { weeks } \\
\text { of pregnancy }\end{array}$ & $\mathrm{LMWH}^{*}$ \\
\hline 2 & 38 & 20.4 & No & No & 3 & $\begin{array}{l}\text { PE during twin pregnancy ended } \\
\text { with IUFD ( } 22 \text { weeks) }\end{array}$ & None \\
\hline 3 & 40 & 35.9 & PTm heterozygous & No & 3 & $\begin{array}{l}\text { DVT in the right leg at } 18 \text { weeks } \\
\text { of pregnancy }\end{array}$ & None \\
\hline
\end{tabular}

*Started when pregnancy test was positive.

BMI, body mass index; DVT, deep vein thrombosis; LMWH, low-molecular-weight heparin; IUFD, intrauterine fetal death; PE, pulmonary embolism; OHSS, ovarian hyperstimulation syndrome; PTm, prothrombin mutation; SVT, superficial vein thrombosis.

prescribed LMWH with/without low-dose aspirin (35 years, range 27-42 years) and the remaining ones (34 years, range $23-46$ years).

Among 234 successful cycles, $10(4.3 \%)$ were complicated by OHSS. An antithrombotic treatment with LMWH was prescribed because of a diagnosis of OHSS in 3 of 23 (13\%) treated cases; in the remaining cases, the indication of an antithrombotic treatment was not indicated by Fertility Centres. On the other hand, an OHSS was recorded in 7 of 211 (3.3\%) untreated pregnancies.

A cohort of 3339 women (median age 31 years, range: 14-46, no significant difference vs cases) who consecutively gave birth at our Hospital after spontaneous conception from January 2010 to December 2012, used as a reference population, showed no significant difference in maternal age and number of live births in respect of the general population of women who delivered between January 2008 and December 2010 in the same geographical area (Apulia region) (table 3). In this reference cohort, a total of 11 vein thromboses were observed: 6 deep vein thrombosis and 5 SVT (table 4). The two-tailed Fisher exact test showed a trend towards statistical significance (p: 0.06 , OR: $3.9,95 \%$ CI 0.87 to 15.3). After the exclusion of superficial thromboses in both the groups, we found that the incidence of VTE in our population of women who had undergone ART was 2/234 pregnancies $(8.5 \%$ ), whereas that in our reference population delivering in our hospital was $6 / 3339$ $(1.8 \% 0)$ (p: 0.09). Furthermore, when we excluded those women who had had a previous VTE (patients 2 and 9 of the reference cohort, table 4), the two-tailed Fisher exact test approached statistical significance (p: 0.054; OR: 7.2, 95\% CI 0.91 to 45.6 ).

\section{DISCUSSION}

To the best of our knowledge, this is the first attempt in Italy to calculate VTE incidence in pregnancies after ART as compared to the risk of pregnancies after natural conception. We found a slightly higher VTE incidence in pregnancies after ART than in those after natural conception. Our findings, although showing only a trend towards statistical significance, most likely due to the small sample size, are in agreement with those recently obtained in other Countries. ${ }^{5-7}$ However, the higher first trimester VTE incidence recorded in previous studies was not confirmed by the present findings; in fact, two of three VTE occurred after the end of the 14th gestational week (table 2).

During the ovulation induction, a haemoconcentration occurs, which with high oestrogen levels and an increased number of small ovarian follicles can predispose to the development of OHSS, which is an important trigger for VTE. ${ }^{8}$

In our study, the role of other potential risk factors, such as the type of drugs (ie, gonadotropins) used for ovulation induction, was not evaluated. The role of inherited and acquired thrombophilias, well established conditions predisposing to thrombosis, seems to be rather weak for pregnant women after ART, as only one of them showed a common cause of thrombophilia (PTm). On the other hand, among the remaining 23 thrombophilic women, none developed a VTE; no VTE was recorded among women carrying the most severe thrombophilias $(n=3)$. We cannot conclude about a potential benefit of antithrombotic prophylaxis in the group carrying thrombophilia, due to the small sample size.

Table 3 Age and live births in the reference cohort and general population from the same geographical area

\begin{tabular}{|c|c|c|c|c|c|}
\hline & \multicolumn{4}{|c|}{ Maternal age } & \multirow{2}{*}{$\begin{array}{l}\text { Live births } \\
\text { number }\end{array}$} \\
\hline & 15 years & 20-29 years & 30-39 years & 40 years & \\
\hline $\begin{array}{l}\text { Reference cohort }(n=3339), \% \\
2010-2012\end{array}$ & 3.7 & 34.4 & 55.1 & 6.8 & 3451 \\
\hline $\begin{array}{l}\text { General population from the same } \\
\text { geographical area }(n=106265), \% \\
2008-2010\end{array}$ & 2.3 & 32.4 & 59.5 & 5.8 & 107461 \\
\hline
\end{tabular}


Table 4 Characteristics of women from the reference cohort experiencing venous thrombosis

\begin{tabular}{|c|c|c|c|c|c|c|}
\hline Patient & $\begin{array}{l}\text { Age at } \\
\text { event }\end{array}$ & BMI & Thrombophilia & Additional risk factors & Type of event & $\begin{array}{l}\text { Antithrombotic } \\
\text { prophylaxis }\end{array}$ \\
\hline 1 & 31 & 37.1 & No & No & Antepartum SVT in the left leg & None \\
\hline 2 & 33 & 18.3 & No & $\begin{array}{l}\text { Previous SVT after } \\
\text { Caesarean section }\end{array}$ & Antepartum DVT in the left leg & None \\
\hline 3 & 28 & 17.3 & FVL heterozygous & Family history for VTE & $\begin{array}{l}\text { Antepartum ( } 37 \text { weeks) DVT in } \\
\text { the right leg }\end{array}$ & $\mathrm{LMWH}^{*} \dagger$ \\
\hline 4 & 33 & NA & NA & No & Postpartum SVT in the right leg & None \\
\hline 5 & 37 & NA & NA & No & Postpartum bilateral SVT & None \\
\hline 6 & 43 & NA & NA & No & Antepartum DVT in the left leg & None \\
\hline 7 & 22 & NA & NA & No & Postpartum DVT in the left leg & None \\
\hline 8 & 35 & NA & NA & No & Postpartum bilateral SVT & None \\
\hline 9 & 36 & 21.3 & $\begin{array}{l}\text { FVL heterozygous+ } \\
\text { PC deficiency }\end{array}$ & $\begin{array}{l}\text { Previous DVT during } \\
\text { oral contraceptive }\end{array}$ & $\begin{array}{l}\text { Antepartum ( } 21 \text { weeks) DVT in } \\
\text { the left leg }\end{array}$ & $\mathrm{LMWH}^{\star} \ddagger$ \\
\hline 10 & 33 & 18.7 & FVL heterozygous & No & Antepartum DVT in the left leg & None \\
\hline 11 & 35 & NA & NA & No & Postpartum SVT in the right leg & None \\
\hline
\end{tabular}

*Started when pregnancy test was positive.

†Treatment withdrawn for physician's choice at 32 weeks.

$\ddagger$ Treatment withdrawn for patient's choice at 20 weeks.

BMI, body mass index; DVT, deep vein thrombosis; FVL, factor V Leiden LMWH, low-molecular-weight heparin; NA, not available;

PC, protein C; PE, pulmonary embolism; SVT, superficial vein thrombosis.

Overall, 10 (4.3\%) OHSS were recorded; this percentage is not significantly different from that reported in other series (15); 3 of 10 OHSS were treated with LMWH, as suggested by the current guidelines, ${ }^{9} 1819$ while the remaining seven cases were not treated; we are not able to investigate the reason for the lack of treatment, but we can hypothesise that they were moderate or mild forms of OHSS. Some limitations of our study need to be considered. First, there is a lack of detailed data on lifestyle factors such as smoking, obesity and thrombophilic status in the reference cohort; it was not possible to obtain these data, as they were extracted from an administrative database. Therefore, we were not able to compare for all possible variables the two groups of women. However, the reference cohort was found to be representative of the general population from the same geographical area, as maternal age and the number of live births were comparable in the two populations. Second, we cannot exclude that in our reference cohort the number of vein thromboses may be underrepresented in respect of the general pregnant population, as some cases may not be admitted to the hospital. However, this possible bias is also present in our group of outpatients; in addition, the incidence we recorded in our reference cohort was found to be similar to that expected in the general population of pregnant women (1: 1000)..$^{12}$ Third, we cannot exclude that during the quite long period of observation (2002-2011) the ART practice has changed; however, it is likely that the number of adverse events is reduced, as improvements were seen in maternal and neonatal outcomes. ${ }^{20}$ On the other hand, the improvement of standard care allowed for a substantial fall in the rate of VTE in general pregnant population. The lack of confounders' control is another limitation and depends on the observational nature of the study and the sample size.

In conclusion, an increased, although not significant, absolute risk of vein thrombosis during pregnancies after ART was found. Further studies aimed at identifying women who could potentially benefit from an antithrombotic prophylaxis are needed.

Acknowledgements The authors acknowledge the women who agreed to participate in this study.

Contributors EG, FD and MM designed the study. MV and GLT enrolled the cases and analysed the data. MV, GLT and EG wrote the manuscript. DC and PV obtained biochemical and molecular results. TP and FP produced data regarding the reference cohort. WA, MM and EG critically revised the manuscript.

Funding The study was supported by the Italian Ministry of Health (Ricerca Corrente 2014).

Competing interests None declared.

Patient consent Obtained.

Ethics approval S. Giovanni Rotondo Ethis Commettee.

Provenance and peer review Not commissioned; externally peer reviewed.

Open Access This is an Open Access article distributed in accordance with the Creative Commons Attribution Non Commercial (CC BY-NC 4.0) license, which permits others to distribute, remix, adapt, build upon this work noncommercially, and license their derivative works on different terms, provided the original work is properly cited and the use is non-commercial. See: http:// creativecommons.org/licenses/by-nc/4.0/

\section{REFERENCES}

1. Lindqvist $P$, Dahlbäck $B$, Marsál K. Thrombotic risk during pregnancy: a population study. Obstet Gynecol 1999;94:595-9.

2. Jacobsen AF, Skjeldestad FE, Sandset PM. Incidence and risk patterns of venous thromboembolism in pregnancy and puerperium - a register-based case-control study. Am J Obstet Gynecol 2008;198:233.e1-7. 
3. Chan WS, Dixon ME. The "ART" of thromboembolism: a review of assisted reproductive technology and thromboembolic complications. Thromb Res 2008;121:713-26.

4. Hansen AT, Kesmodel US, Juul S, et al. No evidence that assisted reproduction increases the risk of thrombosis: a Danish national cohort study. Hum Reprod 2012;27:1499-503.

5. Hansen AT, Kesmodel US, Juul S, et al. Increased venous thrombosis incidence in pregnancies after in vitro fertilization. Hum Reprod 2014;29:611-17.

6. Rova K, Passmark H, Lindqvist PG. Venous thromboembolism in relation to in vitro fertilization: an approach to determining the incidence and increase in risk in successful cycles. Fertil Steril 2012:97:95-100.

7. Henriksson $\mathrm{P}$, Westerlund $\mathrm{E}$, Wallén $\mathrm{H}$, et al. Incidence of pulmonary and venous thromboembolism in pregnancies after in vitro fertilisation: cross sectional study. BMJ 2013;346:e8632.

8. Chan WS. The 'ART' of thrombosis: a review of arterial and venous thrombosis in assisted reproductive technology. Curr Opin Obstet Gynecol 2009;21:207-18.

9. Jenkins JM, Drakeley AJ, Mathur RS. The management of ovarian hyperstimulation syndrome. In: Green-top guideline no. 5. London: Royal College of Obstetricians and Gynecologists, 2006.

10. Grandone E, Colaizzo D, Vergura P, et al. Age and homocysteine plasma levels are risk factors for thrombotic complications after ovarian stimulation. Hum Reprod 2004;19:1796-9.

11. Ricci G, Bogatti P, Fischer-Tamaro L, et al. Factor V Leiden and prothrombin gene G20210A mutation and in vitro fertilization: prospective cohort study. Hum Reprod 2011;26:3068-77.

12. Zegers-Hochschild F, Adamson GD, de Mouzon J, et al, International Committee for Monitoring Assisted Reproductive Technology; World Health Organization. The International Committee for Monitoring Assisted Reproductive Technology
(ICMART) and the World Health Organization (WHO) Revised Glossary on ART Terminology, 2009. Hum Reprod 2009;24:2683-7.

13. Verlato $P$, Zucchetta $P$, Prandoni $P$, et al. An unexpectedly high rate of pulmonary embolism in patients with superficial thrombophlebitis of the thigh. J Vasc Surg 1999;30:1113-15.

14. http://www.salute.gov.it/imgs/c_17_pubblicazioni_1551_allegato.pdf; http://www.salute.gov.it/imgs/C_17 pubblicazioni_1731_allegato.pdf http://www.salute.gov.it/imgs/c_17_pubblicazioni_2024_allegato.pdf

15. Miyakis S, Lockshin MD, Atsumi T, et al. International consensus statement on an update of the classification criteria for definite antiphospholipid syndrome (APS). J Thromb Haemost 2006;4:295-306.

16. Grandone E, Colaizzo D, Tiscia G, et al., PRENACEL Study Group. Impact of common thrombophilias and JAK2 V617F on pregnancy outcomes in unselected Italian women. J Thromb Haemost 2011;9:496-501.

17. Villani M, Tiscia GL, Margaglione M, et al. Risk of obstetric and thromboembolic complications in family members of women with previous adverse obstetric outcomes carrying common inherited thombophilias. J Thromb Haemost 2012;10:223-8.

18. Royal College of Obstetricians and Gynecologists. Reducing the risk of thrombosis and embolism during pregnancy and the puerperium. London: RCOG, 2007 (Guideline no 37a).

19. Bates SM, Greer IA, Middeldorp S, et al., American College of Chest Physicians. VTE, thrombophilia, antithrombotic therapy, and pregnancy: Antithrombotic Therapy and Prevention of Thrombosis, 9th ed: American College of Chest Physicians Evidence-Based Clinical Practice Guidelines. Chest 2012;141(2 Suppl): e691S-736S.

20. Finnström O, Källen B, Lindam A, et al. Maternal and child outcome after in vitro fertilization-a review of 25 years of population-based data from Sweden. Acta Obstet Gynecol Scand 2011;90:494-500. 